\title{
Antigen Processing and Presentation: TAPping into ABC Transporters
}

\section{Citation}

Procko, Erik, and Rachelle Gaudet. Forthcoming. Antigen processing and presentation: TAPping into $A B C$ transporters. Current Opinion in Immunology.

\section{Published Version}

http://dx.doi.org/10.1016/j.coi.2009.02.003

\section{Permanent link}

http://nrs.harvard.edu/urn-3:HUL.InstRepos:2624506

\section{Terms of Use}

This article was downloaded from Harvard University's DASH repository, and is made available under the terms and conditions applicable to Other Posted Material, as set forth at http:// nrs.harvard.edu/urn-3:HUL.InstRepos:dash.current.terms-of-use\#LAA

\section{Share Your Story}

The Harvard community has made this article openly available.

Please share how this access benefits you. Submit a story.

Accessibility 


\title{
Antigen processing and presentation: TAPping into ABC transporters
}

Erik Procko and Rachelle Gaudet

Department of Molecular and Cellular Biology, Harvard University, 7 Divinity Ave, Cambridge MA 02138, USA

Address correspondence to gaudet@mcb.harvard.edu

Running title: TAP function and mechanism

\begin{abstract}
Adaptive, cell-mediated immunity involves the presentation of antigenic peptides on class I MHC molecules at the cell surface. This requires an $\mathrm{ABC}$ transporter associated with antigen processing (TAP) to transport antigenic peptides generated in the cytosol into the endoplasmic reticulum (ER) for loading onto class I MHC. Recent crystal structures of bacterial ABC transporters suggest how the transmembrane domains of TAP form a peptide-binding cavity that acquires peptides from the cytosol, and following ATP-induced conformational changes, the peptide-binding cavity closes to the cytosol and instead opens to the ER lumen for peptide release. Extensive biochemical studies show how transport is driven by ATP binding and hydrolysis on an asymmetric pair of cytosolic nucleotide-binding domains, which are physically coupled to the peptidebinding site to propagate conformational changes through the protein.
\end{abstract}




\section{Introduction}

A central component of immune surveillance is the recognition of foreign antigenic peptides presented by class I MHC molecules on the surface of infected or otherwise aberrant cells (see [1] for a recent review). Most of these peptides are derived from intracellular proteins turned over and degraded in the cytosol. These peptides are recognized by $\mathrm{T}$ lymphocytes when bound to class I MHC molecules at the cell surface, and hence the cytosolic peptides must cross a membrane to gain access to the extracellular environment. This compartmentalization problem is solved during class I MHC processing and assembly in the endoplasmic reticulum (ER) by an ATP-driven engine dedicated to peptide transport. The transporter associated with antigen processing (TAP), a heterodimer of homologous TAP1 and TAP2 subunits, uses the energy of ATP binding and hydrolysis to transport diverse peptides from the cytosol into the ER lumen $[2,3]$. In the ER membrane, TAP, chaperones and class I MHC molecules form a large peptide loading complex (PLC) that co-localizes peptide transport and loading onto MHC molecules [4]. Previous reviews have summarized much of the biochemical and functional data on TAP (e.g. $[5,6])$. In this review, after briefly describing the peptide loading complex, we will focus on the structure and mechanism of its keystone, the TAP transporter.

\section{The peptide-loading complex}

When intracellular proteins are turned over by ubiquitin-proteasome mediated degradation, short peptides are generated, some of which are transported by TAP into the ER lumen for loading on to class I MHC molecules (Figure 1A; see [7] for a recent 
review). Prior to peptide loading, class I heavy chain and $\beta_{2}$-microglobulin are assembled via interactions with protein disulfide isomerases (PDI), and the lectin-type chaperones calnexin and calreticulin [7]. Calreticulin-bound class I MHC heterodimers are then recruited into a large peptide-loading complex (PLC) that contains TAP at its core $[4,8]$. Additional chaperones join the PLC, including ERp57/ER60 (officially classified as PDIA3; protein disulfide isomerase family A, member 3) and the class Ispecific chaperone tapasin. PLC architecture remains controversial [7-9], and further analysis is required to clarify the stoichiometry of components and possible dynamic changes as peptides are transported and bound to MHC. Tapasin interacts with the key components of the PLC: its C-terminal transmembrane and cytosolic region interacts with the TAP subunits [10,11], and its ER lumenal domains interact with class I MHC [11]. Tapasin facilitates class I MHC loading [12], at least in part by forming a disulfide-linked complex with ERp57, an oxidoreductase that assists appropriate disulfide bond formation [13]. This disulfide bond between the two chaperones sequesters ERp57 activity and prevents the enzyme from reducing a critical disulfide bond in the class I MHC peptidebinding groove [14]. After class I MHC has bound an antigenic peptide, ER-resident chaperones are released, allowing the peptide-bound MHC to migrate through the Golgi apparatus and onwards to the cell surface.

At the heart of the peptide-loading complex is the TAP transporter. TAP is a member of the large and ubiquitous family of ATP-binding cassette (ABC) transporters, which pump diverse substrates across various cellular membranes. All ABC transporters share a common modular architecture of two transmembrane domains (TMDs) and two 
nucleotide-binding domains (NBDs) arranged from one or more polypeptide chains. The simplest transporters are assembled from identical subunits and hence are symmetric with two identical TMDs and NBDs, whereas the more complex eukaryotic transporters tend to have non-identical domains, often fused at the genetic level in a single polypeptide. TAP is a heterodimer of homologous but non-equivalent TAP1 and TAP2 subunits, which each provide a single TMD and NBD [15-17]. The two TMDs and two NBDs of $\mathrm{ABC}$ transporters form a core unit capable of substrate transport, but are often supplemented by accessory domains that provide additional functions, such as regulation, substrate capture, or binding of associated proteins [18]. Both TAP1 and TAP2 have an additional transmembrane, N-terminal accessory domain that binds tapasin during PLC assembly [19-21], although these domains are not required for the fundamental task of peptide transport $[19,21]$.

\section{A shared general mechanism for $\mathrm{ABC}$ transporters}

All ABC transporters likely share a common mechanism (see [22] for a recent review). The two TMDs form a cavity for substrate binding that alternates between facing one side of the membrane for substrate acquisition and the other side for substrate release (Figure 1B). This alternating access mechanism is driven by ATP binding to the cytosolic NBDs and subsequent ATP hydrolysis (Figure 1B). Each NBD binds an ATP molecule, and the two ATP-bound NBDs form a closed, tight association [23]. The ATP molecules are clasped at the interface and provide contacts to both NBDs [24]. When the NBDs are closed around ATP, the TMDs form an outward-facing cavity ('outward' is defined as the side of the membrane opposite the cytosolic NBDs) [25-28]. Following ATP hydrolysis, 
the NBDs open and the substrate-binding cavity of the TMDs faces inwards $[27,29,30]$ (Figure 1B). Importers and exporters use the same basic set of conformations, but differ on which states bind the substrate with high or low affinity.

There are now multiple crystal structures of bacterial ABC transporters, both importers and exporters, and in inward- and outward-facing conformations [25-30]. Two of these bacterial transporters have significant homology to TAP and can therefore provide templates for three-dimensional models: a putative drug exporter (Sav1866; [28]) and a lipid flippase (MsbA) that has been crystallized in multiple conformations (some likely non-physiological) [27]. The Sav1866 and MsbA structures illustrate the arrangement of the four core domains: two TMDs and two NBDs (Figure 2A). Each TMD consists of a bundle of six long helices that extend into the cytoplasm. Unexpectedly, the TMDs show domain-swapping: helices 1 and 2 of one polypeptide chain bundle with helices 3-6 of the second polypeptide and vice-versa, so that the both polypeptide chain participate in the formation of each bundle of transmembrane helices. The NBDs form the closed, ATPdependent dimer previously observed in structures of isolated NBDs (Figure 2B), and interact with the TMDs by a short coupling helix (Figure 2). The implications of this coupling helix are discussed below.

\section{The TAP peptide binding site}

Various strategies have been used to map residues in TAP that contact antigenic peptide, including chemically active peptides that cleave or cross-link to TAP [31] and the identification of a polymorphic site that alters TAP's peptide specificity $[32,33]$. When 
these peptide-binding residues are mapped onto the corresponding residues of Sav1866, they cluster at the base of an outward-facing cavity (Figure 2A; [28]). Structural models of TAP, based on the Sav1866 and MsbA structures, support the hypothesis that this putative peptide-binding cavity alternates between facing the cytosolic and ER lumenal sides of the membrane as the NBDs open and close [34]. While such models suggest where peptides bind TAP, the exact mechanism by which TAP selects and binds peptide substrates is still unknown, although the peptide specificity of TAP is well-established ([35] and references therein). Class I MHC molecules present peptides of 8-10 residues with specific amino acids at anchor positions to provide strong contacts between the peptide and $\mathrm{MHC}$ molecule, whereas at other positions the peptide sequence is free to vary. TAP must similarly bind peptides in a manner that provides sufficient affinity but allows peptide sequence diversity. Human TAP prefers peptides with basic or hydrophobic amino acids at the C-terminus, which complements MHC specificity, and has a weaker preference for basic residues near the N-terminus. These preferences presumably reflect the chemical nature of critical sites on TAP that grasp the peptide substrate. Hence both TAP and MHC display specificity for certain amino acids at a few positions in the peptide substrate to provide affinity, but also recognize a huge diversity of peptide sequences to allow presentation of many different epitopes to the immune system. However, TAP favors 8-13-residue peptides, slightly longer than the canonical 8-10 amino acids that fit onto class I MHC. These longer peptides are trimmed at their N-termini by the ER-associated aminopeptidase (ERAP; [36-38]), producing the required length for class I MHC binding and increasing peptide sequence diversity at the Nterminus. 


\section{Asymmetry in the TAP ATPase sites refines a model for peptide transport}

As described above, ATP-dependent NBD closure and subsequent ATP hydrolysis-driven opening of the NBDs energize the conformational changes in the membrane-spanning regions of TAP required for transport (Figure 1B). Upon NBD closure, two composite ATPase sites are formed at the interface between the TAP1- and TAP2-NBDs (Figure 2B). The ATPase sites include characteristic motifs found in all ABC transporters and many other ATPases [24]. Each NBD first binds ATP via interactions with the Walker A motif which contacts the $\alpha$ - and $\beta$-phosphates and coordinates an ATP-associated magnesium ion, the Walker B motif which contains a critical acidic residue that positions and polarizes the hydrolytic water molecule, and the switch motif which contacts the labile $\gamma$-phosphate. The second NBD can now interact with this ATP-bound site and complete the active site by contributing the D-loop which positions and polarizes the hydrolytic water via a backbone carbonyl, and the signature motif which contacts the $\gamma$ phosphate and mediates ATP-dependent NBD association. Only in the composite active site of the closed NBD dimer are the hydrolytic water molecule and $\gamma$-phosphate appropriately positioned for hydrolysis [24,39]. High resolution crystal structures of TAP1-NBD, both as an ADP-bound monomer [40] and as an ATP-bound dimer used as a surrogate model for the physiological TAP1-/TAP2-NBD dimer [39], support this mechanism.

Unlike most bacterial ABC transporters, some motifs for ATP binding and hydrolysis show departures from consensus in both TAP1 and TAP2, such that in the context of an 
ATP-bound TAP dimer, one ATPase site has consensus motifs, while the second ATPase site has substitutions away from consensus [39]. The two ATPase sites are referred to as the consensus and degenerate sites, respectively. Within the degenerate site, substitutions in the switch and Walker B motifs markedly reduce ATPase activity, while substitutions in the signature motif weaken ATP-dependent NBD association [39]. Because the degenerate site is impaired, the consensus site is the principal driver of NBD closure and ATP hydrolysis. This is supported by multiple studies that introduce damaging mutations into the TAP ATPase sites; mutations are partially tolerated in the degenerate site, but peptide transport activity is lost when the equivalent mutations are introduced in the consensus site [41-44]. This property of having only one consensus ATPase site, while the second site has acquired substitutions that impair its activities, is common amongst eukaryotic $\mathrm{ABC}$ transporters. A similar phenomenon is seen in other families of oligomeric ATPases, where homo-oligomeric bacterial ATPases with consensus motifs have evolved into complex hetero-oligomers with full activity retained in only a subset of active sites. The implications of the evolution toward heterogeneous active sites are not fully understood. It may simply be that the impairment of one ATPase site is tolerated in heterodimers. Alternatively, it may facilitate more complex functions and regulatory mechanisms.

The TAP1- and TAP2-NBDs also have unique nucleotide specificities. TAP1-NBD binds both ATP and ADP, similar to most ABC transporter NBDs, whereas the TAP2NBD has a strong preference for ADP $([42,45]$ and E. Procko, A. McFedries and R. Gaudet, unpublished data). However, at some stage during the transport cycle, TAP2 
binds and hydrolyzes ATP, which is critical for driving conformational changes associated with transport [46] - TAP2 forms the main contacts to the ATP hydrolyzed in the consensus ATPase site [39]. While it has been suggested that TAP2 only displays a preference for ADP when isolated from TAP1 and that the physiological TAP $1 / 2$ heterodimer has no such nucleotide preferences [47], another possibility is that the nucleotide specificity of TAP2 is regulated during a transport cycle. Using chimeras between the TAP1 and TAP2 proteins, it was observed that a short stretch of amino acids near the C-terminus is responsible for the unique nucleotide specificities [48]. In the TAP1-NBD structure $[39,40]$, these amino acids are distant from the ATP-binding site and instead form a hinge-like loop that bridges two subdomains (Figure 2B). The NBD fold comprises two lobes, an ATPase subdomain and a helical subdomain, that are capable of rigid body rotations. While a crystal structure of TAP2-NBD has yet to be determined, the length and residues of the hinge element differ in TAP1 and TAP2. We hence hypothesize that the distinct nucleotide specificities of TAP1 and TAP2 are due to different relative orientations of the NBD subdomains, encoded by their unique hinge regions. In crystal structures of full-length bacterial ABC transporters, a short helical element from each TMD, called a coupling helix, contacts each respective NBD [22]. This coupling helix fits in a groove between the two NBD subdomains such that TMD motions could direct the NBD subdomains into different orientations (Figure 2). The domain swapping observed in the structure of the Sav1866 TMDs described above, if conserved in TAP, would entail an interaction of the TAP1 coupling helix with the TAP2 NBD and vice-versa, possibly explaining how TAP1 seems to alter the nucleotidebinding preference of TAP2 [47]. This influence of the coupling helix on nucleotide- 
binding specificity could also be dynamic. For example, antigenic peptide binding to the TMDs could cause a conformational change transmitted, via the coupling helix, to the TAP2-NBD, altering its subdomain orientations to promote ADP-ATP exchange or NBD closure.

We recently proposed a model for the mechanism of TAP illustrated in Figure 1B [34,39]. The transport cycle of TAP begins in an inward-facing conformation with the NBDs apart. The binding of peptide substrate to the cytosolic-facing cavity of the TMDs causes a conformational change that enables NBD closure, perhaps by facilitating exchange of ADP for ATP on TAP2. ATP-driven NBD closure is coupled to TMD motions that flip the peptide-binding cavity to an outward/lumenal-facing conformation. This leads to peptide release, as supported by biochemical experiments indicating that TAP has lowest affinity for peptides when the NBDs are trapped in a closed state [31]. ATP hydrolysis in the consensus ATPase site (possibly followed by a nonessential hydrolysis of ATP in the degenerate site) causes the NBDs to re-open, resetting the inward-facing conformation for another transport cycle.

\section{Viral inhibitors of TAP}

A number of viruses target TAP to subvert class I MHC presentation and evade immune surveillance. $\mathrm{mK} 3$ of murine gammaherpesvirus- 68 and UL49.5 of bovine herpesvirus 1 (BHV-1) bind and target TAP for proteasomal degradation $[49,50]$. Other viral inhibitors arrest TAP in a translocation-incompetent state. US6 of human cytomegalovirus (HCMV) and UL49.5 of equine herpesvirus (EHV) types 1 and 4 lock TAP in a state that 
can still bind antigenic peptides, but block the interaction of TAP with ATP [51-56]. US6, which interacts with TAP on the ER lumenal side, could propagate its inhibition to the cytosolic nucleotide-binding sites of TAP via the coupling helices' proposed modulation of NBD subdomain orientation. The UL49.5 proteins of BHV-1 and pseudorabies virus (PRV) arrest TAP in a state that can still bind peptides without preventing ATP interactions [49,51]. Finally, the cytosolic ICP47 proteins of herpes simplex viruses (HSV) block the TAP peptide-binding cavity by competing with antigenic peptides [57,58]. Therefore these viral inhibitors - US6, UL49.5 and ICP47 likely all trap TAP in an inward-facing, open-NBDs conformation, blocking the initiation of a transport cycle (Figure 3). This is reminiscent of substrate-mediated trans-inhibition observed for some bacterial $\mathrm{ABC}$ importers, in which the transported substrate binds a cytosolic regulatory domain to lock the transporter in the inward-facing, open-NBD conformation when cytoplasmic substrate levels are high $[59,60]$. The viral TAP inhibitors may function analogously to the regulatory domains of these importers, and by blocking the transporter prior to ATP-driven NBD closure the inhibitors would avoid working against the energy provided by ATP hydrolysis to dissociate the NBDs. A possible exception to this general inhibition mechanism may be presented by the BNLF2a protein of Epstein-Barr virus and its close relatives. BNLF2a interacts with and locks TAP in a conformation that is unable to bind free ATP or peptides [61]. The nucleotideand peptide-binding sites may simply be occluded, or alternatively BNLF2a may lock TAP in an outward-facing, closed-NBD conformation, in which the now lumenal-facing peptide-binding cavity has low substrate affinity and the closed ATPase sites are inaccessible for nucleotide exchange. Additional viral inhibitors of this important 
transporter for antigen presentation will likely be discovered and will continue to be useful tools to determine the molecular mechanism of peptide transport by TAP.

\section{Concluding remarks}

Recent bacterial $\mathrm{ABC}$ transporter structures have advanced our understanding of the TAP transporter, how it functions in the peptide loading complex, and how it is inhibited by viral proteins. In turn, TAP has proven to be an excellent model for asymmetric $\mathrm{ABC}$ transporters because it is readily amenable to biochemical experimentation and several viral inhibitors of TAP function are available. We anticipate that this reciprocal interaction between the antigen presentation and $\mathrm{ABC}$ transporter fields will continue to yield insights into $\mathrm{ABC}$ transporter function in antigen presentation and beyond.

\section{Acknowledgments}

We apologize to those whose work we could not cite due to space constraints. The authors thank lab members for discussions and advice. Work in RG's group is partly funded by the American Cancer Society (RSG GMC-111847) and EP was supported by a Merck-Wiley Fellowship. 


\section{Figure legends}

\section{Figure 1. Antigenic peptide transport and loading}

A. Nascent class I MHC heavy chains and $\beta_{2}$-microglobulin are assembled with assistance from numerous chaperones (a). The MHC heterodimer is then recruited into the TAP-containing PLC via interactions with tapasin (b). Peptides generated by ubiquitin-proteasome mediated protein turnover (c), some subsequently trimmed by cytosolic peptidases [62], are transported by TAP from the cytosol into the ER lumen (d). These peptides are bound by class I MHC, some after further trimming in the ER by ERAP, causing the fully folded class I MHC-peptide complexes to shed ER-associated chaperones (e). The peptide-MHC molecules migrate to the cell surface (f and g) where they are scanned by $\mathrm{CD} 8^{+} \mathrm{T}$ cells. B. A model of peptide transport begins with TAP in an inward/cytosol-facing conformation with the NBDs open. A peptide binds a cytosolfacing cavity formed by the TMDs, causing a conformational change that is transmitted to the NBDs. This conformational change permits ATP-dependent NBD closure, perhaps by facilitating exchange of ADP on TAP2 for ATP. As the NBDs close, the peptidebinding cavity closes to the cytosol and opens to the ER lumen, creating the closed, outward-facing conformation of TAP. Peptide affinity is markedly reduced in this conformation, and the peptide is hence released into the ER. ATP hydrolysis in the consensus ATPase site is sufficient to destabilize the closed NBDs (hydrolysis may also occur in the degenerate ATPase site, but this is not essential), which re-open to generate the resting conformation. 


\section{Figure 2. Structural information on TAP}

A. Ribbon representation of the Sav1866 crystal structure, a TAP homologue from bacteria, with closed NBDs and an outward-facing cavity. The cavity (indicated with a cone) has a roughly V-shaped opening that faces the top of the structure in this orientation. The approximate position of the membrane is shaded blue. Positions equivalent to those that bind antigenic peptides in TAP are indicated with colored spheres, including a polymorphic site that alters TAP peptide specificity (blue; $[32,33]$ ), a site that is cleaved by reactive peptides (yellow; [31]), and a site that can cross-link to cysteine-containing peptides (red; [31]). These positions are near the base of the cavity, and are predicted to be accessible for peptide binding when the conformation changes to expose the cavity to the inside/cytosol. The coupling helices from the TMDs that interact with the NBDs are shown as solid cylinders. B. Homology model of TAP1-NBD bound to TAP2-NBD in the closed conformation with ATP at the interface [63]. Each composite ATPase site is formed by Walker A (dark blue), Walker B (purple) and switch (red) motifs from one NBD, and signature (yellow) and D-loop (cyan) motifs from the second NBD, highlighted on the top, degenerate ATPase site. The NBD fold has ATPase and helical subdomains (labeled for TAP2), and a coupling helix $(\mathrm{CH}$, represented with a cylinder) from a TMD fits in the groove between the two subdomains, such that the TMD conformation could alter the subdomain orientations. The hinge elements that determine the unique nucleotide-binding specificities of TAP1 and TAP2, possibly by setting the preferred subdomain orientations, are colored brown. 


\section{Figure 3. Viral inhibition of TAP}

Several viral proteins inhibit TAP by arresting the transporter in the resting conformation, with the NBDs open and the peptide-binding cavity facing the cytosol. HSV ICP47 competitively blocks the peptide-binding site (A), UL49.5 of BHV-1 and PRV arrest the transporter (B), and HCMV US6 prevents interactions with ATP while still permitting ADP binding (C; the UL49.5 proteins of EHV-1/4 also prevent ATP binding). These viral inhibitors arrest TAP analogously to substrate-mediated trans-inhibition of certain bacterial $\mathrm{ABC}$ transporters, in which the transported substrate binds cytosolic regulatory domains to lock the transporter in the inward-facing, open-NBDs conformation. Shown is the crystal structure of a trans-inhibited tungstate/molybdate transporter (ModBC) [59], with tungstate ions bound to the cytosolic regulatory domains (D). 


\section{Key papers}

27. Ward A, Reyes CL, Yu J, Roth CB, Chang G: Flexibility in the ABC transporter MsbA: Alternating access with a twist. Proc Natl Acad Sci $U S A$ 2007, 104:19005-19010.

28. Dawson RJ, Locher KP: Structure of a bacterial multidrug ABC transporter. Nature 2006, 443:180-185.

-• The crystal structures of Sav1866 and MsbA, two bacterial ABC transporters distantly related to TAP, reveal a putative substrate-binding cavity formed by transmembrane helices. The transmembrane helices are physically coupled to the NBDs, providing a mechanism for how conformational changes are propagated between the NBDs and substrate-binding cavity.

\section{Herget M, Oancea G, Schrodt S, Karas M, Tampe R, Abele R: Mechanism of} substrate sensing and signal transmission within an $\mathrm{ABC}$ transporter: use of a Trojan horse strategy. J Biol Chem 2007, 282:3871-3880.

-• TAP residues in proximity to bound peptide substrates were mapped using reactive peptides that could cross-link to or cleave the TAP protein. It was also shown that TAP has its highest affinity for peptides in the absence of nucleotides, and peptide affinity is lowest following treatment with $\mathrm{ADP} \cdot \mathrm{AlF}_{\mathrm{X}}$, which could trap the TAP NBDs in the closed, dimeric conformation.

\section{Procko E, Ferrin-O'Connell I, Ng SL, Gaudet R: Distinct structural and} functional properties of the ATPase sites in an asymmetric ABC transporter. Mol Cell 2006, 24:51-62.

-• Using an ATP-dependent TAP1-NBD homodimer as a model system for the physiological TAP1-NBD/TAP2-NBD dimer, it was shown that the degenerate ATPase site has reduced catalytic activity and poorly stabilizes the ATP-bound NBD dimer. Mutations in full-length TAP showed a single consensus site is sufficient for transport.

\section{Perria CL, Rajamanickam V, Lapinski PE, Raghavan M: Catalytic site} modifications of TAP1 and TAP2 and their functional consequences. $J$ Biol Chem 2006, 281:39839-39851.

- Mutations were introduced into TAP to prevent hydrolysis in the consensus ATPase site, and peptide transport was lost. However, mutagenesis of the degenerate ATPase site had only a small effect, supporting the hypothesis that ATP hydrolysis chiefly occurs in the consensus site.

48. Ehses S, Leonhardt RM, Hansen G, Knittler MR: Functional role of C-terminal sequence elements in the transporter associated with antigen processing. $J$ Immunol 2005, 174:328-339.

- By making chimeras between the TAP1 and TAP2 polypeptides, it was demonstrated that a small sequence element which links the two NBD subdomains determines the unique nucleotide specificity of TAP2, namely that TAP2 has a strong preference for ADP over ATP. 


\section{Gerber S, Comellas-Bigler M, Goetz BA, Locher KP: Structural Basis of Trans-}

Inhibition in a Molybdate/tungstate ABC Transporter. Science 2008.

60. Kadaba NS, Kaiser JT, Johnson E, Lee A, Rees DC: The high-affinity E. coli methionine $\mathrm{ABC}$ transporter: structure and allosteric regulation. Science 2008, 321:250-253.

- The crystal structures of two bacterial ABC transporters, MetNI and ModBC, demonstrate a mechanism by which $\mathrm{ABC}$ transporters can be conformationally locked to inhibit transport. When cytoplasmic levels of the imported substrate are already high, the substrate can bind to cytosolic regulatory domains of MetNI or ModBC to trap the transporter with open NBDs and an inward-facing substrate-binding cavity.

61. Hislop AD, Ressing ME, van Leeuwen D, Pudney VA, Horst D, Koppers-Lalic D, Croft NP, Neefjes JJ, Rickinson AB, Wiertz EJ: A CD8+ T cell immune evasion protein specific to Epstein-Barr virus and its close relatives in Old World primates. $J$ Exp Med 2007, 204:1863-1873.

- The authors identify the BNLF2a protein of Epstein-Barr virus and its close relatives as a TAP inhibitor that prevents binding of ATP or peptides to TAP. This possibly represents a new mechanism of TAP inhibition by viral proteins. 


\section{References}

1. Godfrey DI, Rossjohn J, McCluskey J: The fidelity, occasional promiscuity, and versatility of $\mathbf{T}$ cell receptor recognition. Immunity 2008, 28:304-314.

2. Kleijmeer MJ, Kelly A, Geuze HJ, Slot JW, Townsend A, Trowsdale J: Location of MHC-encoded transporters in the endoplasmic reticulum and cis-Golgi. Nature 1992, 357:342-344.

3. Androlewicz MJ, Anderson KS, Cresswell P: Evidence that transporters associated with antigen processing translocate a major histocompatibility complex class I-binding peptide into the endoplasmic reticulum in an ATP-dependent manner. Proc Natl Acad Sci U S A 1993, 90:9130-9134.

4. Ortmann B, Androlewicz MJ, Cresswell P: MHC class I/beta 2-microglobulin complexes associate with TAP transporters before peptide binding. Nature 1994, 368:864-867.

5. Momburg F, Hengel H: Corking the bottleneck: the transporter associated with antigen processing as a target for immune subversion by viruses. Curr Top Microbiol Immunol 2002, 269:57-74.

6. Abele R, Tampe R: The ABCs of immunology: structure and function of TAP, the transporter associated with antigen processing. Physiology (Bethesda) 2004, 19:216-224.

7. Wearsch PA, Cresswell P: The quality control of MHC class I peptide loading. Curr Opin Cell Biol 2008.

8. Rufer E, Leonhardt RM, Knittler MR: Molecular architecture of the TAP-associated MHC class I peptide-loading complex. J Immunol 2007, 179:5717-5727.

9. Ortmann B, Copeman J, Lehner PJ, Sadasivan B, Herberg JA, Grandea AG, Riddell SR, Tampe R, Spies T, Trowsdale J, et al.: A critical role for tapasin in the assembly and function of multimeric MHC class I-TAP complexes. Science 1997, 277:1306-1309.

10. Bangia N, Lehner PJ, Hughes EA, Surman M, Cresswell P: The N-terminal region of tapasin is required to stabilize the MHC class I loading complex. Eur $J$ Immunol 1999, 29:1858-1870.

11. Lehner PJ, Surman MJ, Cresswell P: Soluble tapasin restores MHC class I expression and function in the tapasin-negative cell line .220. Immunity 1998, 8:221-231.

12. Zarling AL, Luckey CJ, Marto JA, White FM, Brame CJ, Evans AM, Lehner PJ, Cresswell $\mathrm{P}$, Shabanowitz J, Hunt DF, et al.: Tapasin is a facilitator, not an editor, of class I MHC peptide binding. J Immunol 2003, 171:5287-5295.

13. Dick TP, Bangia N, Peaper DR, Cresswell P: Disulfide bond isomerization and the assembly of MHC class I-peptide complexes. Immunity 2002, 16:87-98.

14. Kienast A, Preuss M, Winkler M, Dick TP: Redox regulation of peptide receptivity of major histocompatibility complex class I molecules by ERp57 and tapasin. Nat Immunol 2007, 8:864-872.

15. Kelly A, Powis SH, Kerr LA, Mockridge I, Elliott T, Bastin J, Uchanska-Ziegler B, Ziegler A, Trowsdale J, Townsend A: Assembly and function of the two ABC transporter proteins encoded in the human major histocompatibility complex. Nature 1992, 355:641-644. 
16. Bahram S, Arnold D, Bresnahan M, Strominger JL, Spies T: Two putative subunits of a peptide pump encoded in the human major histocompatibility complex class II region. Proc Natl Acad Sci U S A 1991, 88:10094-10098.

17. Spies T, Cerundolo V, Colonna M, Cresswell P, Townsend A, DeMars R: Presentation of viral antigen by MHC class I molecules is dependent on a putative peptide transporter heterodimer. Nature 1992, 355:644-646.

18. Biemans-Oldehinkel E, Doeven MK, Poolman B: ABC transporter architecture and regulatory roles of accessory domains. FEBS Lett 2006, 580:1023-1035.

19. Koch J, Guntrum R, Heintke S, Kyritsis C, Tampe R: Functional dissection of the transmembrane domains of the transporter associated with antigen processing (TAP). J Biol Chem 2004, 279:10142-10147.

20. Koch J, Guntrum R, Tampe R: The first N-terminal transmembrane helix of each subunit of the antigenic peptide transporter TAP is essential for independent tapasin binding. FEBS Lett 2006, 580:4091-4096.

21. Procko E, Raghuraman G, Wiley DC, Raghavan M, Gaudet R: Identification of domain boundaries within the N-termini of TAP1 and TAP2 and their importance in tapasin binding and tapasin-mediated increase in peptide loading of MHC class I. Immunol Cell Biol 2005, 83:475-482.

22. Hollenstein K, Dawson RJ, Locher KP: Structure and mechanism of ABC transporter proteins. Curr Opin Struct Biol 2007, 17:412-418.

23. Moody JE, Millen L, Binns D, Hunt JF, Thomas PJ: Cooperative, ATP-dependent association of the nucleotide binding cassettes during the catalytic cycle of ATP-binding cassette transporters. J Biol Chem 2002, 277:21111-21114.

24. Smith PC, Karpowich N, Millen L, Moody JE, Rosen J, Thomas PJ, Hunt JF: ATP binding to the motor domain from an $\mathrm{ABC}$ transporter drives formation of a nucleotide sandwich dimer. Mol Cell 2002, 10:139-149.

25. Locher KP, Lee AT, Rees DC: The E. coli BtuCD structure: a framework for ABC transporter architecture and mechanism. Science 2002, 296:1091-1098.

26. Oldham ML, Khare D, Quiocho FA, Davidson AL, Chen J: Crystal structure of a catalytic intermediate of the maltose transporter. Nature 2007, 450:515-521.

27. Ward A, Reyes CL, Yu J, Roth CB, Chang G: Flexibility in the ABC transporter MsbA: Alternating access with a twist. Proc Natl Acad Sci U S A 2007, 104:19005-19010.

28. Dawson RJ, Locher KP: Structure of a bacterial multidrug ABC transporter. Nature 2006, 443:180-185.

29. Pinkett HW, Lee AT, Lum P, Locher KP, Rees DC: An inward-facing conformation of a putative metal-chelate-type $\mathbf{A B C}$ transporter. Science 2007, 315:373-377.

30. Hollenstein K, Frei DC, Locher KP: Structure of an ABC transporter in complex with its binding protein. Nature 2007, 446:213-216.

31. Herget M, Oancea G, Schrodt S, Karas M, Tampe R, Abele R: Mechanism of substrate sensing and signal transmission within an $\mathrm{ABC}$ transporter: use of a Trojan horse strategy. $J$ Biol Chem 2007, 282:3871-3880.

32. Deverson EV, Leong L, Seelig A, Coadwell WJ, Tredgett EM, Butcher GW, Howard JC: Functional analysis by site-directed mutagenesis of the complex 
polymorphism in rat transporter associated with antigen processing. $J$ Immunol 1998, 160:2767-2779.

33. Momburg F, Armandola EA, Post M, Hammerling GJ: Residues in TAP2 peptide transporters controlling substrate specificity. J Immunol 1996, 156:1756-1763.

34. Procko E, O'Mara ML, Bennett WFD, Tieleman P, Gaudet R: The mechanism of ABC transporters: general lessons from structural and functional studies of an antigenic peptide transporter. FASEB Journal 2008, In press.

35. Schmitt L, Tampe R: Affinity, specificity, diversity: a challenge for the $\mathbf{A B C}$ transporter TAP in cellular immunity. Chembiochem 2000, 1:16-35.

36. Serwold T, Gonzalez F, Kim J, Jacob R, Shastri N: ERAAP customizes peptides for MHC class I molecules in the endoplasmic reticulum. Nature 2002, 419:480483.

37. Saric T, Chang SC, Hattori A, York IA, Markant S, Rock KL, Tsujimoto M, Goldberg AL: An IFN-gamma-induced aminopeptidase in the ER, ERAP1, trims precursors to MHC class I-presented peptides. Nat Immunol 2002, 3:1169-1176.

38. York IA, Chang SC, Saric T, Keys JA, Favreau JM, Goldberg AL, Rock KL: The ER aminopeptidase ERAP1 enhances or limits antigen presentation by trimming epitopes to 8-9 residues. Nat Immunol 2002, 3:1177-1184.

39. Procko E, Ferrin-O'Connell I, Ng SL, Gaudet R: Distinct structural and functional properties of the ATPase sites in an asymmetric $\mathrm{ABC}$ transporter. $\mathrm{Mol} \mathrm{Cell}$ 2006, 24:51-62.

40. Gaudet R, Wiley DC: Structure of the ABC ATPase domain of human TAP1, the transporter associated with antigen processing. Embo $J$ 2001, 20:4964-4972.

41. Perria CL, Rajamanickam V, Lapinski PE, Raghavan M: Catalytic site modifications of TAP1 and TAP2 and their functional consequences. $J$ Biol Chem 2006, 281:39839-39851.

42. Alberts P, Daumke O, Deverson EV, Howard JC, Knittler MR: Distinct functional properties of the TAP subunits coordinate the nucleotide-dependent transport cycle. Curr Biol 2001, 11:242-251.

43. Lapinski PE, Neubig RR, Raghavan M: Walker A lysine mutations of TAP1 and TAP2 interfere with peptide translocation but not peptide binding. $\mathrm{J}$ Biol Chem 2001, 276:7526-7533.

44. Karttunen JT, Lehner PJ, Gupta SS, Hewitt EW, Cresswell P: Distinct functions and cooperative interaction of the subunits of the transporter associated with antigen processing (TAP). Proc Natl Acad Sci U S A 2001, 98:7431-7436.

45. Bouabe H, Knittler MR: The distinct nucleotide binding states of the transporter associated with antigen processing (TAP) are regulated by the nonhomologous C-terminal tails of TAP1 and TAP2. Eur J Biochem 2003, 270:4531-4546.

46. Chen M, Abele R, Tampe R: Peptides induce ATP hydrolysis at both subunits of the transporter associated with antigen processing. $J$ Biol Chem 2003, 278:29686-29692.

47. Lapinski PE, Raghuraman G, Raghavan M: Nucleotide interactions with membrane-bound transporter associated with antigen processing proteins. $J$ Biol Chem 2003, 278:8229-8237. 
48. Ehses S, Leonhardt RM, Hansen G, Knittler MR: Functional role of C-terminal sequence elements in the transporter associated with antigen processing. $J$ Immunol 2005, 174:328-339.

49. Koppers-Lalic D, Reits EA, Ressing ME, Lipinska AD, Abele R, Koch J, Marcondes Rezende M, Admiraal P, van Leeuwen D, Bienkowska-Szewczyk K, et al.: Varicelloviruses avoid $T$ cell recognition by UL49.5-mediated inactivation of the transporter associated with antigen processing. Proc Natl Acad Sci U S A 2005, 102:5144-5149.

50. Boname JM, de Lima BD, Lehner PJ, Stevenson PG: Viral degradation of the MHC class I peptide loading complex. Immunity 2004, 20:305-317.

51. Koppers-Lalic D, Verweij MC, Lipinska AD, Wang Y, Quinten E, Reits EA, Koch J, Loch S, Rezende MM, Daus F, et al.: Varicellovirus UL 49.5 proteins differentially affect the function of the transporter associated with antigen processing, TAP. PLoS Pathog 2008, 4:e1000080.

52. Ahn K, Gruhler A, Galocha B, Jones TR, Wiertz EJ, Ploegh HL, Peterson PA, Yang Y, Fruh K: The ER-luminal domain of the HCMV glycoprotein US6 inhibits peptide translocation by TAP. Immunity 1997, 6:613-621.

53. Kyritsis C, Gorbulev S, Hutschenreiter S, Pawlitschko K, Abele R, Tampe R:

Molecular mechanism and structural aspects of transporter associated with antigen processing inhibition by the cytomegalovirus protein US6. $J$ Biol Chem 2001, 276:48031-48039.

54. Hengel H, Koopmann JO, Flohr T, Muranyi W, Goulmy E, Hammerling GJ, Koszinowski UH, Momburg F: A viral ER-resident glycoprotein inactivates the MHC-encoded peptide transporter. Immunity 1997, 6:623-632.

55. Lehner PJ, Karttunen JT, Wilkinson GW, Cresswell P: The human cytomegalovirus US6 glycoprotein inhibits transporter associated with antigen processingdependent peptide translocation. Proc Natl Acad Sci U S A 1997, 94:69046909.

56. Hewitt EW, Gupta SS, Lehner PJ: The human cytomegalovirus gene product US6 inhibits ATP binding by TAP. Embo J 2001, 20:387-396.

57. Ahn K, Meyer TH, Uebel S, Sempe P, Djaballah H, Yang Y, Peterson PA, Fruh K, Tampe R: Molecular mechanism and species specificity of TAP inhibition by herpes simplex virus ICP47. Embo $J$ 1996, 15:3247-3255.

58. Tomazin R, Hill AB, Jugovic P, York I, van Endert P, Ploegh HL, Andrews DW, Johnson DC: Stable binding of the herpes simplex virus ICP47 protein to the peptide binding site of TAP. Embo $J$ 1996, 15:3256-3266.

59. Gerber S, Comellas-Bigler M, Goetz BA, Locher KP: Structural Basis of TransInhibition in a Molybdate/tungstate ABC Transporter. Science 2008.

60. Kadaba NS, Kaiser JT, Johnson E, Lee A, Rees DC: The high-affinity E. coli methionine $\mathrm{ABC}$ transporter: structure and allosteric regulation. Science 2008, 321:250-253.

61. Hislop AD, Ressing ME, van Leeuwen D, Pudney VA, Horst D, Koppers-Lalic D, Croft NP, Neefjes JJ, Rickinson AB, Wiertz EJ: A CD8+ T cell immune evasion protein specific to Epstein-Barr virus and its close relatives in Old World primates. $J$ Exp Med 2007, 204:1863-1873. 
62. Kloetzel PM, Ossendorp F: Proteasome and peptidase function in MHC-class-Imediated antigen presentation. Curr Opin Immunol 2004, 16:76-81.

63. Procko E, Gaudet R: Functionally important interactions between the nucleotidebinding domains of an antigenic peptide transporter. Biochemistry 2008, 47:5699-5708. 


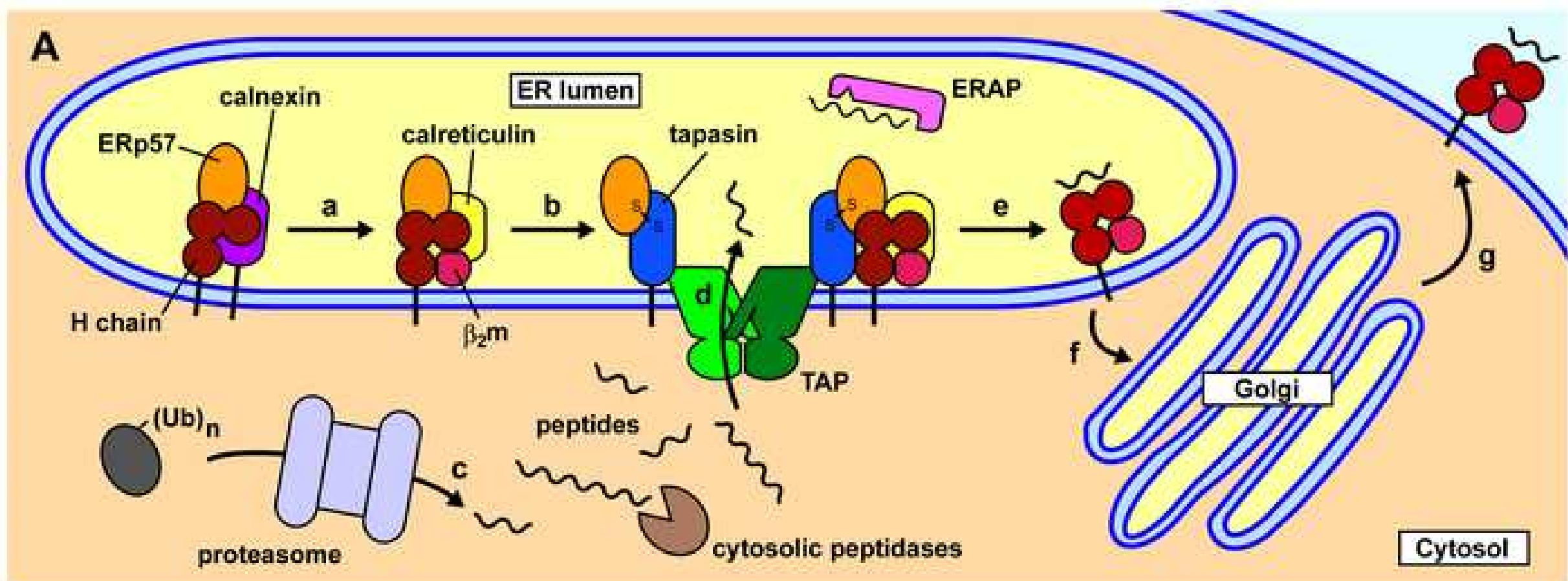

B

NBD opening resets the inward-facing conformation

ER lumen

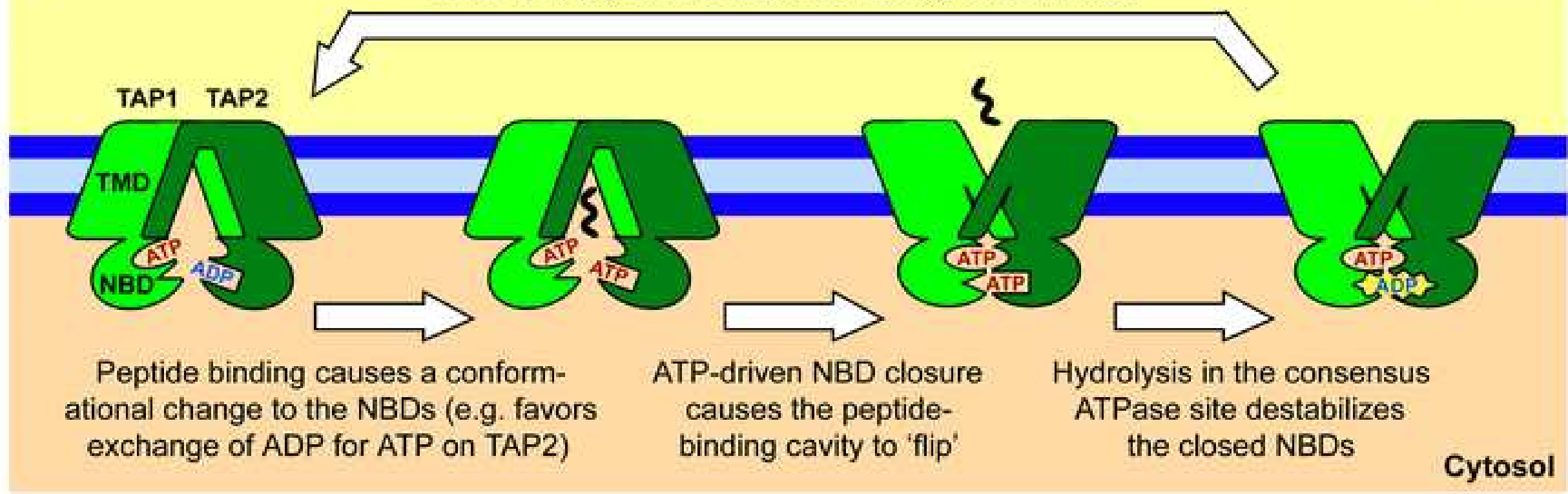


A

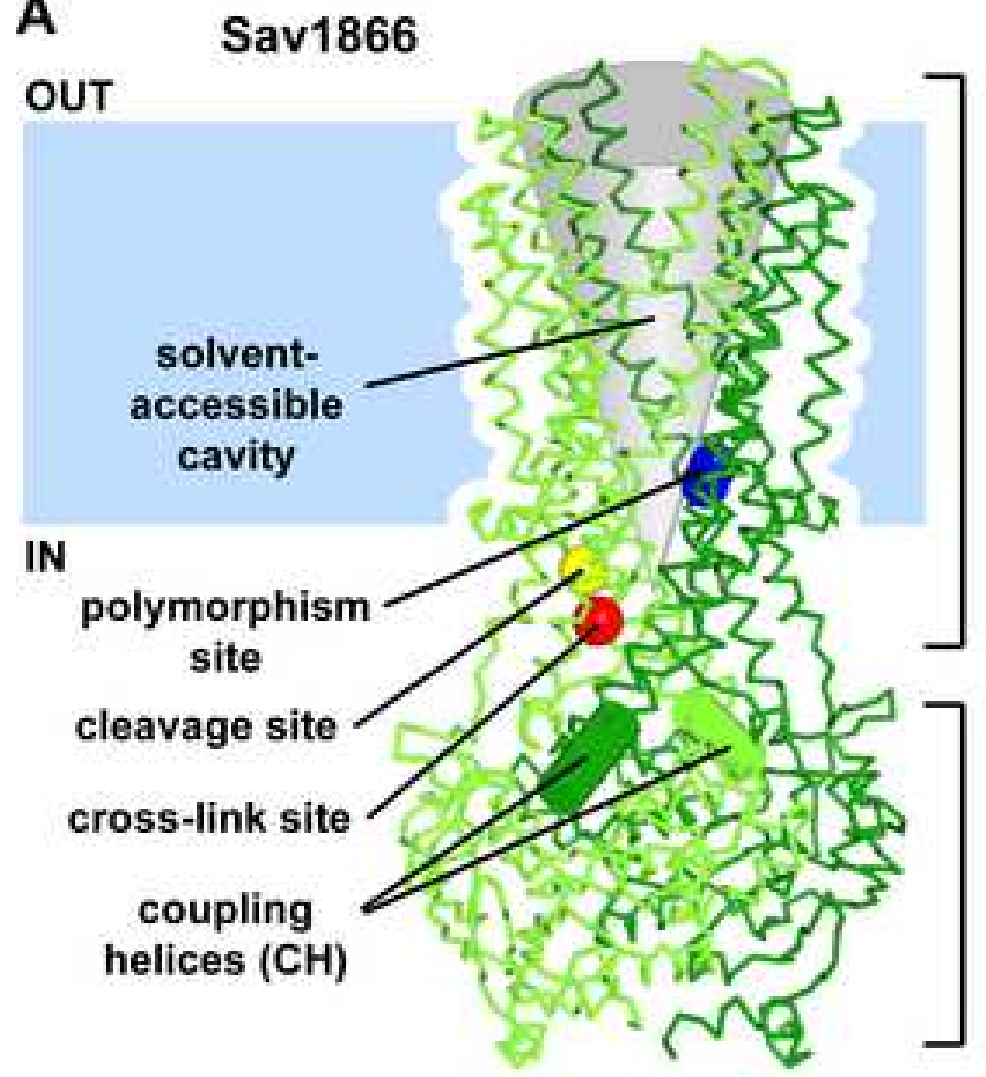

B

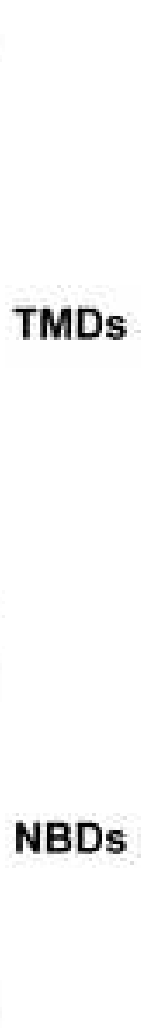

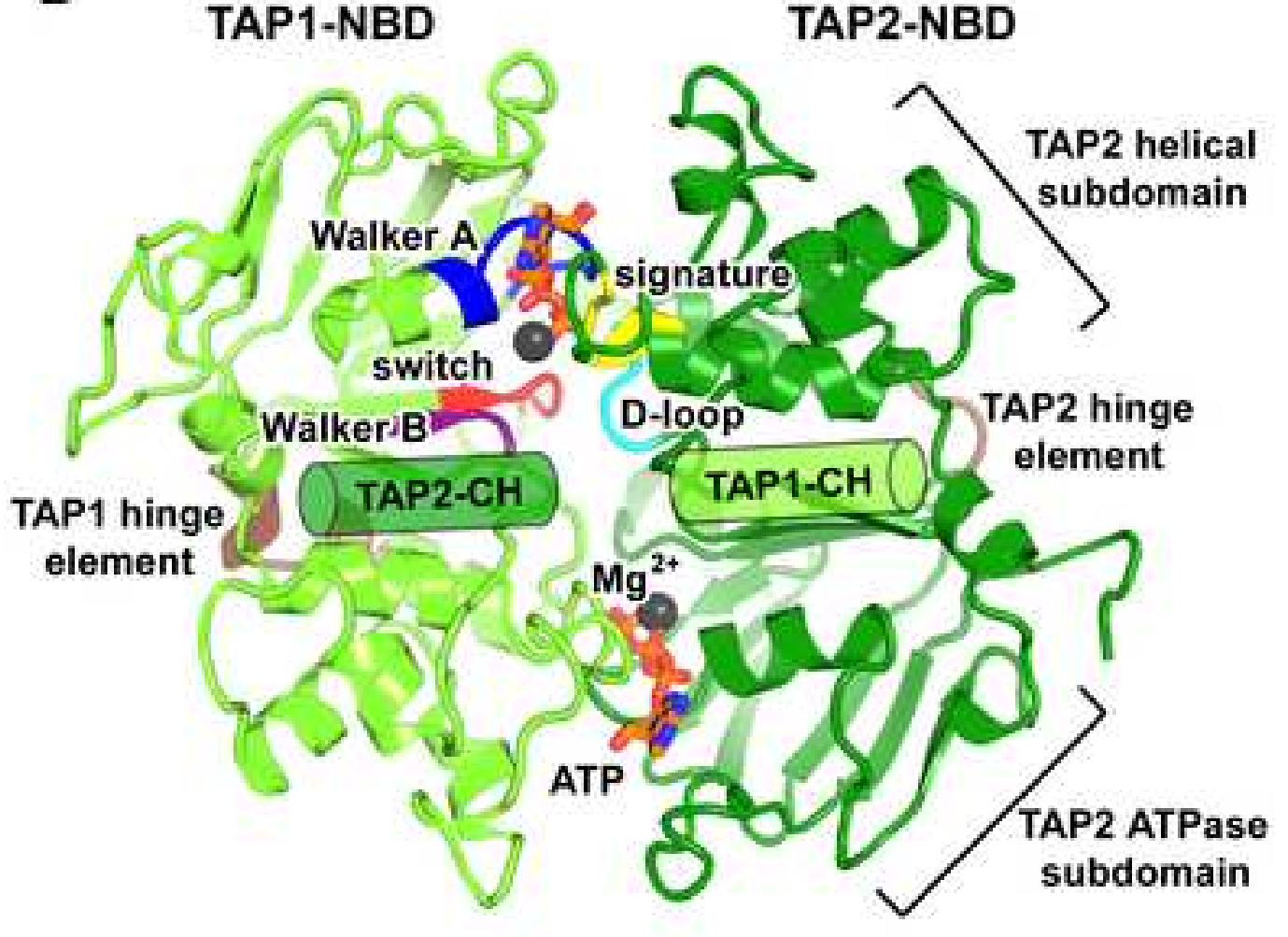




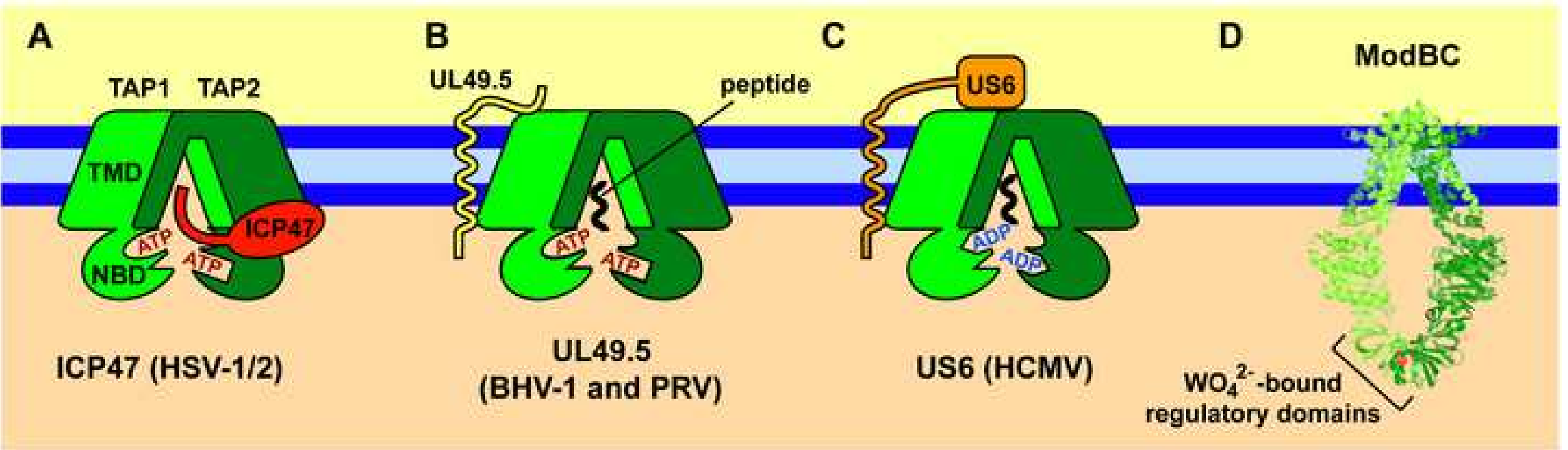

